\title{
Thermoconvective instability of paramagnetic fluids in a uniform magnetic field
}

\author{
Jie Huang and Boyd F. Edwards \\ Department of Physics, West Virginia University, P.O. Box 6315, Morgantown, West Virginia 26506-6315
}

Donald D. Gray

Department of Civil and Environmental Engineering, West Virginia University, P.O. Box 6103, Morgantown, West Virginia 26506-6103

(Received 13 November 1996; accepted 18 February 1997)

\begin{abstract}
The effect of a uniform oblique magnetic field on a laterally unbounded insulating paramagnetic fluid layer heated from below is studied using a linear stability analysis of the Navier-Stokes equations supplemented by Maxwell's equations and the appropriate magnetic body force. Two-dimensional rolls in an arbitrary vertical plane are considered. Longitudinal rolls with axes parallel to the horizontal component of the field are the rolls most unstable to convection. The corresponding critical Rayleigh number and critical wavelength for the onset of such rolls are less than the well-known Rayleigh-Bénard values in the absence of magnetic fields. Vertical fields maximize these deviations, which vanish for horizontal fields. Horizontal fields increase the critical Rayleigh number and the critical wavelength for all rolls except longitudinal rolls. These effects should be observable in careful experiments at high fields. (C) 1997 American Institute of Physics.
\end{abstract} [S1070-6631(97)02106-5]

\section{INTRODUCTION}

An infinitely extended horizontal layer of fluid heated from below is the classical geometry for the study of buoyancy-driven convective instabilities. ${ }^{1,2}$ In this classical problem, the fluid is driven by maintaining the bottom of the fluid layer at a temperature $\Delta T$ higher than the top. Thermal expansion renders the mass density $\rho$ lower near the bottom than near the top; the resulting gradient in the gravitational force per unit volume $\mathbf{f}_{g}=\rho \mathbf{g}$ thereby renders the fluid potentially unstable. For small $\Delta T$, the fluid remains at rest. Above a critical value $\Delta T_{c}$, buoyancy is strong enough to overcome the dissipative effects of viscosity and thermal conduction, and convection begins. The convective instability is controlled by the Rayleigh number $R$, which measures the strength of buoyancy relative to dissipation,

$$
R=\frac{\alpha g \Delta T d^{3}}{D_{T} \nu}
$$

where $\alpha$ is the thermal expansion coefficient, $g$ the acceleration of gravity, $D_{T}$ the thermal diffusivity, $\nu$ the kinematic viscosity, and $d$ the thickness of the layer. Under the Oberbeck-Boussinesq approximation, which allows small density gradients only in the large gravity term of the Navier-Stokes equations, the convection occurs above a critical Rayleigh number $R=R_{0 c} \approx 1708$ for rigid (no-slip) boundary conditions. This universal number is independent of the fluid properties.

We are interested in studying how uniform applied magnetic fields modify this classical scenario for paramagnetic fluids, which are subject to the Kelvin body force, ${ }^{3}$ whose density is $\mathbf{f}_{m}=\mu_{0} M \nabla H$. This force arises from the interaction between the local magnetic field $\mathbf{H}$ (of magnitude $H$ ) within the fluid and the molecular magnetic moments characterized by the magnetization $\mathbf{M}$ (of magnitude $M$ ), the magnetic moment per unit volume. Here, $\mu_{0}$ is the permeability of free space. This force tends to move paramagnetic fluids toward regions of higher magnetic field. For typical paramagnetic fluids, the magnetization satisfies $\mathbf{M}=\chi \mathbf{H}$, with a Curie volumetric susceptibility ${ }^{4} \chi=C \rho / T$ that is proportional to the ratio of the mass density $\rho$ to the temperature $T$, where $C$ is a constant characteristic of the fluid.

We consider a horizontal paramagnetic fluid layer of thickness $d$ defined by $|z|<d / 2$, with $\hat{z}$ pointing up. A vertical thermal gradient is imposed on the layer. We consider two-dimensional convective rolls in the $x-z$ plane with the axes of these rolls parallel to the $y$ axis. This layer is subjected to a uniform external magnetic field $\mathbf{H}_{0}$ $=H_{0} \cos \phi \cos \xi \hat{x}+H_{0} \cos \phi \sin \xi \hat{y}+H_{0} \sin \phi \hat{z}$ directed at an angle $\phi$ with respect to the horizontal. Here, $\xi$ represents the azimuthal angle between the vertical plane containing $\mathbf{H}_{\mathbf{0}}$ and the $x-z$ plane. The roll axes are perpendicular to the horizontal component of the field for $\xi=0$ ("transverse rolls"), and are parallel to this component for $\xi=\pi / 2$ ("'longitudinal rolls"). In the absence of magnetic fields or in the presence of a vertical uniform magnetic field $\left(\mathbf{H}_{0}=H_{0} \hat{z}\right)$, the layer is rotationally symmetric about its $z$ axis. However, this symmetry is broken when the layer is placed in an inclined field ( $\cos \phi \neq 0)$.

Elementary electromagnetism easily yields the magnetic field and the Kelvin body force for a static fluid layer. In the absence of perturbations of the magnetic field outside the layer due to convection inside the layer, the magnetic field, 


$$
\mathbf{H}= \begin{cases}H_{0} \cos \phi \cos \xi \hat{x}+H_{0} \cos \phi \sin \xi \hat{y}+H_{0} \sin \phi \hat{z}, & |z|>d / 2, \\ H_{0} \cos \phi \cos \xi \hat{x}+H_{0} \cos \phi \sin \xi \hat{y}+\frac{H_{0}}{(1+\chi)} \sin \phi \hat{z}, & |z|<d / 2,\end{cases}
$$

follows simply by demanding continuity of the normal component of magnetic induction $\mathbf{B} \equiv \mu_{0}(\mathbf{M}+\mathbf{H})$ and of the tangential component of $\mathbf{H}$ at $z= \pm d / 2$. This local magnetic field satisfies Maxwell's equations exactly. Imposing a downward thermal gradient by heating from below yields an upward gradient in the magnetic susceptibility $\chi=C \rho / T$ and a corresponding downward gradient in $H$. Ignoring here any density variations with temperature, the Kelvin force density follows easily as

$$
\mathbf{f}_{m}=\mu_{0} H_{0}^{2} \sin ^{2} \phi \frac{\chi^{2}}{(1+\chi)^{3}} \frac{\boldsymbol{\nabla} T}{T} \sim \sin ^{2} \phi \frac{\boldsymbol{\nabla} T}{T^{3}} .
$$

The second form for $\mathbf{f}_{m}$ emphasizes the dependences on $\phi$ and $T$ for small typical susceptibilities $\chi \approx 10^{-3}$. Clearly, this $\mathbf{f}_{m}$ for the static layer is in the same direction as the thermal gradient and has a magnitude that decreases with increasing temperature. Thus, $\mathbf{f}_{\mathbf{m}}$ is directed downward for a fluid layer heated from below, and is strongest near the top of the layer. This force therefore acts to destabilize such a layer in a manner similar to the gravitational body force density $\mathbf{f}_{g}=\rho \mathbf{g}$. In contrast with $\mathbf{f}_{g}$, however, $\mathbf{f}_{m}$ also tends to destabilize a fluid layer that is heated from above. A striking feature of this force is that it is invariant under $\phi \rightarrow-\phi$; $\mathbf{f}_{m}$ is the same whether the magnetic field is directed above or below the horizontal as long as the angle with the horizontal is the same. Furthermore, $\mathbf{f}_{m}$ reaches its maximum for vertical fields with $\phi= \pm \pi / 2$ and vanishes entirely for horizontal fields with $\phi=0$. Hence, vertical applied magnetic fields (either upward or downward) yield the greatest destabilizing effect on the static fluid layer. This destabilizing effect stems from the vertical susceptibility gradient resulting from the imposed vertical thermal gradient due to heating from below.

As will be shown below, uniform vertical applied magnetic fields do indeed tend to destabilize a static paramagnetic fluid layer heated from below, producing lower critical Rayleigh numbers $R_{c}<R_{0 c}$ than in the classical, nonmagnetic case. Thus, uniform vertical magnetic fields promote convection, owing to the vertical gradients in the Curie susceptibility resulting from the imposed thermal gradient. This effect decreases as the applied field begins to tilt toward the horizontal, as expected from the $\sin ^{2} \phi$ dependence of $\mathbf{f}_{m}$.

In this paper, a linear stability analysis of a horizontal paramagnetic fluid layer heated from below in the presence of a uniform oblique magnetic field shows that oscillatory instability cannot occur and longitudinal rolls are the rolls most unstable to convection. In Sec. II, we outline the basic equations and boundary conditions, and present the static state solution. In Sec. III, we summarize the linear equations governing the convective perturbations, and outline the numerical method used to solve these equations. We summarize the main results and draw conclusions in Sec. IV.

\section{EQUATIONS OF MOTION}

For an incompressible magnetic fluid in the presence of a magnetic field, the hydrodynamic momentum equation takes the form ${ }^{3}$

$$
\rho \frac{d \mathbf{V}}{d t}=\rho \mathbf{g}-\nabla p+\rho \nu \nabla^{2} \mathbf{V}+\mathbf{f}_{m},
$$

where the magnetic body force density is

$$
\mathbf{f}_{m}=-\boldsymbol{\nabla}\left[\mu_{0} \int_{0}^{H}\left(\frac{\partial M v}{\partial v}\right)_{H, T} d H\right]+\mu_{0} M \boldsymbol{\nabla} H .
$$

Here $t$ is time, $\mathbf{V}$ the fluid velocity, $d / d t=\partial / \partial t+\mathbf{V} \cdot \boldsymbol{\nabla}$ the material derivative, $v=\rho^{-1}$ the specific volume, and $p$ the pressure. We assume that the viscosity is isotropic and is independent of the magnetic field. The first term in $\mathbf{f}_{m}$ vanishes for Curie paramagnetic fluids since their susceptibilities are proportional to their mass densities $(M \sim 1 / v)$.

Conservation of energy yields the temperature equation for an incompressible paramagnetic fluid that obeys Fourier's law (see the Appendix),

$$
\rho c_{p, \mathbf{H}} \frac{d T}{d t}-\mu_{0} \mathbf{M} \cdot \frac{d \mathbf{H}}{d t}=\kappa \nabla^{2} T+\Phi,
$$

where $c_{p, \mathbf{H}}$ is the specific heat capacity at constant pressure and magnetic field, $\kappa$ is the thermal conductivity (assumed constant), and $\Phi$ is the viscous dissipation.

For an electrically nonconducting fluid, we write the Maxwell's equations,

$$
\boldsymbol{\nabla} \cdot \mathbf{B}=0
$$

and

$$
\boldsymbol{\nabla} \times \mathbf{H}=0 .
$$

The density equation of state is linearized about the average temperature $T_{a}$ of the layer according to

$$
\rho=\rho_{a}\left[1-\alpha\left(T-T_{a}\right)\right] .
$$

The small density variations are ignored throughout this paper except in the term involving gravity, consistent with the Oberbeck-Boussinesq approximation.

The magnetic equation of state is also linearized about $T_{a}$ and an average magnetic field $H_{a}$ to become

$$
M=M_{a}+\chi_{a}\left(H-H_{a}\right)-\kappa_{a}\left(T-T_{a}\right),
$$

where $\chi_{a}=C \rho_{a} / T_{a}$ and $\kappa_{a}=\chi_{a} H_{a} / T_{a}$ are the susceptibility and the pyromagnetic coefficient for a paramagnetic fluid.

We now state the boundary conditions. Equations (5a) and $(5 \mathrm{~b})$ require that the normal component of magnetic induction and the tangential component of magnetic field are continuous across the top and bottom boundaries,

$$
[\hat{z} \cdot \mathbf{B}]_{-}^{+}=0 \text { and }[\hat{z} \times \mathbf{H}]_{-}^{+}=0, \quad \text { at } z= \pm d / 2 \text {. }
$$


Here, $[q]_{-}^{+}=\lim _{\epsilon \rightarrow 0}\left(\left.q\right|_{z= \pm d / 2+\epsilon}-\left.q\right|_{z= \pm d / 2-\epsilon}\right)$ is the difference between the values of a quantity $q$ above and below the boundaries. Rigid boundary conditions require a vanishing velocity,

$$
\mathbf{V}=0, \quad \text { at } z= \pm d / 2,
$$

and the temperature is assumed uniform on each boundary,

$$
T= \begin{cases}T_{0}, & \text { at } z=d / 2, \\ T_{1}, & \text { at } z=-d / 2,\end{cases}
$$

with $T_{a}=\left(T_{0}+T_{1}\right) / 2$.

In the static state, the solution of Eqs. (2)-(8) is

$$
\begin{aligned}
& \mathbf{V}_{s}=0, \quad T_{s}=T_{a}-\beta z, \quad H_{s x}=H_{0} \cos \phi \cos \xi, \\
& H_{s y}=H_{0} \cos \phi \sin \xi,
\end{aligned}
$$

and

$$
H_{s z}=\frac{H_{0} \sin \phi}{1+\chi_{a}}-\frac{\chi_{a} H_{0} \sin \phi \beta z}{\left(1+\chi_{a}\right)^{2} T_{a}},
$$

where $\beta=\left(T_{1}-T_{0}\right) / d=\Delta T / d$ is the thermal gradient. This solution also follows from the field $\mathbf{H}$ obtained in the Introduction by considering $\Delta T / T_{a}$ as small.

\section{EQUATIONS FOR CONVECTIVE STATE}

To investigate convective stability, we add infinitesimal perturbations to the static state, substitute this perturbed state into the governing equations (2)-(7), and linearize these equations to yield the equations governing the perturbations. To write these equations in dimensionless form, we choose $d, d^{2} / D_{T}, \Delta T$, and $\chi_{a} H_{0} \Delta T /\left(1+\chi_{a}\right) T_{a}$ as the scales for length, time, temperature, and magnetic field, respectively. Here, $D_{T} \equiv \kappa / \rho_{a} c_{p, \mathbf{H}}$ is the thermal diffusivity. Finally, we write the linearized dimensionless equations for the convective state,

$$
\begin{aligned}
& \frac{1}{\operatorname{Pr}} \frac{\partial}{\partial t} \nabla^{2} w=\nabla^{4} w+R \nabla_{\perp}^{2} \theta+K \sin ^{2} \phi \nabla_{\perp}^{2} \theta \\
& -K \nabla_{\perp}^{2} \hat{H}_{0} \cdot \nabla \psi, \\
& \frac{\partial \theta}{\partial t}-L \hat{H}_{0} \cdot \frac{\partial \nabla \psi}{\partial t}=\nabla^{2} \theta+\left(1-\frac{L \sin ^{2} \phi}{\left(1+\chi_{a}\right)^{2}}\right) w, \\
& \nabla^{2} \psi-\hat{H}_{0} \cdot \nabla \theta=0,
\end{aligned}
$$

where $w$ is the $z$ component of the velocity perturbation $\mathbf{v}, \theta$ is the temperature perturbation, $\psi$ is the magnetic potential in the field perturbation $\mathbf{h}=\boldsymbol{\nabla} \psi, \hat{H}_{0}=\mathbf{H}_{0} / H_{0}, \quad \nabla_{\perp}^{2} \equiv \partial^{2} / \partial x^{2}$ $+\partial^{2} / \partial y^{2}$, and the Prandtl number $\operatorname{Pr}=\nu / D_{T}$. The two dimensionless parameters $K$, the "Kelvin" number, and $L$ are defined by

$$
K=\frac{\mu_{0} \chi_{a}^{2} H_{0}^{2} \Delta T^{2} d^{2}}{\left(1+\chi_{a}\right) \rho_{a} T_{a}^{2} D_{T} \nu} \quad \text { and } \quad L=\frac{\mu_{0} \chi_{a}^{2} H_{0}^{2}}{\left(1+\chi_{a}\right) T_{a} \rho_{a} c_{p, \mathbf{H}}} .
$$

Here, we use the values of the density and specific heat capacity for water to estimate the typical value for the geometry-independent parameter $L$. A typical value for the volumetric susceptibility of paramagnetic fluids is $\chi_{a}$ $\sim 10^{-3}$. For a magnetic induction $B_{0}=10 \mathrm{~T}$, we have $L$ $\sim 10^{-7} \ll 1$ at room temperature. We accordingly neglect the terms involving $L$ in Eq. (11).

We now study the effects of an inclined magnetic field on linear two-dimensional convective rolls in an arbitrary vertical plane. Since the $x-z$ plane is indeed an arbitrary plane with respect to the field ( $\xi$ is a free parameter), we consider convective rolls in this plane without losing generality. We accordingly write these rolls as

$$
\left\{\begin{array}{c}
w=\left[w_{1}(z) \cos q x+w_{2}(z) \sin q x\right] \exp (\sigma t), \\
\theta=\left[\theta_{1}(z) \cos q x+\theta_{2}(z) \sin q x\right] \exp (\sigma t), \\
\psi=\left[\psi_{1}(z) \cos q x+\psi_{2}(z) \sin q x\right] \exp (\sigma t),
\end{array}\right.
$$

where $q$ is the dimensionless wave number of these perturbations and $\sigma$ the growth rate. Though not listed explicitly here, we also write perturbations of the magnetic field outside the fluid layer induced by the convective motion of the fluid, in the same manner as $\psi$.

The dimensionless boundary conditions are

$$
\begin{aligned}
& w=\frac{\partial w}{\partial z}=\theta=0, \quad \text { at } \quad z= \pm \frac{1}{2}, \\
& \left(1+\chi_{a}\right) \frac{\partial \psi}{\partial z}= \begin{cases}-q \psi, & z=\frac{1}{2}, \\
q \psi, & z=-\frac{1}{2} .\end{cases}
\end{aligned}
$$

We first consider the oscillatory instability of these rolls. Substituting Eq. (13) into Eqs. (10), (11), and (12) yields a set of ordinary differential equations, which can be solved by the Galerkin method. We expand $w_{i}$ according to

$$
w_{i}(z)=\sum_{m} A_{i m} C_{m}(z), \quad \text { for }(i=1,2),
$$

where

$$
C_{m}(z)=\frac{\cosh \left(\lambda_{m} z\right)}{\cosh \left(\lambda_{m} / 2\right)}-\frac{\cos \left(\lambda_{m} z\right)}{\cos \left(\lambda_{m} / 2\right)}
$$

which satisfies $C_{m}(z)=d C_{m} / d z=0$ at $z= \pm 1 / 2$. The numerical values for $\lambda_{m}$ have been tabulated. ${ }^{1}$ We also expand $\theta_{i}$ and $\psi_{i}$ in series of $C_{m}(z)$. A one-term approximation yields

$$
A_{1} \sigma^{2}+A_{2} \sigma+A_{3}=0
$$

where 


$$
\left\{\begin{array}{l}
A_{1}=\left(q^{2}+C_{11}\right)^{2} / \operatorname{Pr} \\
A_{2}=\left(q^{2}+C_{11}\right)\left(\lambda_{1}^{4}+2 C_{11} q^{2}+q^{4}+A_{1}\right) \\
A_{3}=\operatorname{Pr} A_{1}\left(\lambda_{1}^{4}+2 C_{11} q^{2}+q^{4}\right)-\left(R+K \sin ^{2} \phi\right) q^{2}\left(q^{2}+C_{11}\right)+K q^{4} \cos ^{2} \phi \cos ^{2} \xi
\end{array}\right.
$$

Here, $\quad \lambda_{1}=4.73004074 \quad$ and $\quad C_{11} \equiv-\int_{-1 / 2}^{1 / 2} C_{1}(z)$ $\times\left[d^{2} C_{1}(z) / d z^{2}\right] d z=12.302619$. The onset of neutral oscillatory instability requires an imaginary growth rate $\sigma=i \omega$. Since the functions $A_{1}, A_{2}$, and $A_{3}$ are all real, Eq. (16) can be satisfied for $\sigma=i \omega$ only if $A_{2}=0$. Since $A_{2}>0$ for all values of $q$, an oscillatory instability cannot occur in the one-term approximation. By setting $\sigma=0$ and $K=0$, Eq. (16) yields an approximate marginal state $R_{0}(q)$ in the absence of magnetic fields, which has a minimum value $R_{0 c}$ $=1887$ located at $q_{0 c}=3.21$. A numerical calculation involving 360 terms yields $R_{0 c}=1708$ and $q_{0 c}=3.12$, which agree with the well-known critical values $R_{0 c}=1707.762$ and $q_{0 c}=3.117$ for the onset of convection. ${ }^{1}$ Although our proof of a stationary instability is based on a one-term analysis, we believe that an analysis including more terms would not alter this conclusion. Thus we limit consideration to a stationary instability.

With $\partial / \partial t=0$, Eqs. (10), (11), and (12) yield the governing equations for the marginal state. We adopt the algorithm of Stiles and Kagan ${ }^{5}$ to solve these equations numerically. Comparing with the previous algorithm of expanding all variables $w_{i}, \theta_{i}$, and $\psi_{i}$ in a series of $C_{m}(z)$, this algorithm yields more rapid convergence for successive approximations. First, we still expand $w_{i}$ according to Eq. (15). We then write

$\theta_{i}=\sum_{m} A_{i m} \theta_{i m} \quad$ and $\quad \psi_{i}=\sum_{m} A_{i m} \psi_{i m}, \quad$ for $\quad(i=1,2$,$) .$

We substitute Eqs. (13), (15), and (17) into Eqs. (11) and (12), and then solve these equations individually to obtain the general solutions for $\theta_{i m}$ and $\psi_{i m}$. We use the boundary conditions, Eqs. (14), to determine the coefficients involved in these general solutions. Substituting the general solutions into Eq. (10), multiplying by $C_{n}(z)$, and integrating over $\left[-\frac{1}{2}, \frac{1}{2}\right]$ yields

$$
\sum_{m} A_{i m} b_{m n}=0, \quad n=1,2,3, \ldots,
$$

with

$$
\begin{aligned}
b_{m n}= & \left(\lambda_{m}^{+}+\frac{q^{4} R}{\lambda_{m}^{-}}-\frac{q^{4} \lambda_{m}^{+} K^{\prime}}{\lambda_{m}^{-2}}\right) \delta_{m n}-\left(2-\frac{R}{\lambda_{m}^{-}}+\frac{2 q^{4} K^{\prime}}{\lambda_{m}^{-2}}\right) \\
& \times q^{2}\left\langle C_{n} \mid D^{2} C_{m}\right\rangle+\frac{q^{2} \lambda_{m}^{2}}{\exp (q / 2) \lambda_{m}^{-2}}\left[\lambda_{m}^{+}+2 q^{4}+4 \lambda_{m} q^{3}\right. \\
& \left.\times \tanh \left(\frac{\lambda_{m}}{2}\right)\right] K^{\prime}\left\langle C_{n} \mid \cosh q z\right\rangle-\left(R+\frac{1}{4}(2+q) K^{\prime}\right) \\
& \times q^{2} r_{m}\left\langle C_{n} \mid \cosh q z\right\rangle+\frac{1}{2} q^{3} r_{m} K^{\prime}\left\langle C_{n} \mid z \sinh q z\right\rangle,
\end{aligned}
$$

where $K^{\prime} \equiv K\left(\sin ^{2} \phi-\cos ^{2} \phi \cos ^{2} \xi\right), D^{2} \equiv d^{2} / d z^{2}, \lambda_{m}^{+} \equiv \lambda_{m}^{4}$ $+q^{4}, \quad \lambda_{m}^{-} \equiv \lambda_{m}^{4}-q^{4}, \quad r_{m} \equiv 2 \lambda_{m}^{2} / \lambda_{m}^{-} \cosh (q / 2), \quad$ and $\langle f \mid g\rangle$ $\equiv \int_{-1 / 2}^{1 / 2} f g d z$. In obtaining these results, we made use of the fact that $\chi_{a} \ll 1$ for paramagnetic fluids.

\section{RESULTS AND CONCLUSIONS}

Remarkably, all of the $\phi$ and $\xi$ dependences and all of the dependence on the Kelvin number $K$ turn out to appear in the combination

$$
K^{\prime} \equiv K\left(\sin ^{2} \phi-\cos ^{2} \phi \cos ^{2} \xi\right),
$$

in the marginal equations governing the onset of convection, Eqs. (18) and (19). As will be seen below, a positive (negative) $K^{\prime}$ tends to destabilize (stabilize) the static fluid layer with respect to convective perturbations. Since the reduced Kelvin number $K^{\prime}=K$ is always positive for a vertical magnetic field $(|\phi|=\pi / 2)$, the field tends to enhance convection. As this reduced Kelvin number $K^{\prime}=K$ for $|\phi|=\pi / 2$ is independent of the angle $\xi$, the field has the same promoting effect on all rolls, reflecting the rotational symmetry of the layer about vertical. Therefore, the pattern of convection that appears at marginal stability is completely unspecified in the presence of a vertical magnetic field.

In the presence of an inclined magnetic field $(|\phi|$ $<\pi / 2$ ), the field breaks the rotational symmetry of the layer about the $z$ axis; it has different effects on different rolls, as indicated through the term $\cos ^{2} \xi$ in the reduced Kelvin number $K^{\prime}$. For longitudinal rolls $\left(\xi=\pi / 2\right.$ ), Eq. (20) yields $K^{\prime}$ $=K \sin ^{2} \phi \equiv K_{\perp}>0$, which implies that the oblique field always enhances these rolls. For transverse rolls $(\xi=0), K^{\prime}$ $=K\left(\sin ^{2} \phi-\cos ^{2} \phi\right) \equiv K_{\|}$. As $K_{\|}>0$ for $\pi / 2>|\phi|>\pi / 4, K_{\|}$ $=0$ for $|\phi|=\pi / 4$, and $K_{\|}<0$ for $\pi / 4>|\phi|>0$, the applied oblique field enhances these rolls when $\pi / 2>|\phi|>\pi / 4$, has no effect on them when $|\phi|=\pi / 4$, and suppresses them when $\pi / 4>|\phi|>0$. Since $K_{\perp}>K^{\prime}>K_{\|}$for $\pi / 2>\xi>0$, the oblique field for given $\phi$ has the maximum enhancing effect on longitudinal rolls; transverse rolls are the most difficult ones to drive. Thus, longitudinal rolls are the most unstable convective rolls in the presence of oblique magnetic fields; convection when it first sets in appears as longitudinal rolls.

As discussed in the Introduction, the destabilizing effect due to the Kelvin body force equation (1) vanishes for horizontal magnetic fields $(\phi=0)$. Since $K^{\prime}=-K \cos ^{2} \xi$ for a horizontal field, the field has no effect on longitudinal rolls $\left(K^{\prime}=0\right)$, consistent with our discussion. Nevertheless, the field suppresses other rolls $\left(0>K^{\prime}>-K\right)$ and has the maximum inhibiting effect on transverse rolls $\left(K^{\prime}=-K\right)$.

A possible mechanism for this suppression of transverse rolls by a horizontal magnetic field is the cost of aligning the intrinsic paramagnetic molecular moments with the field in the circulating convective flow. (A magnetic moment that is not aligned with the field is subject to a magnetic torque.) 


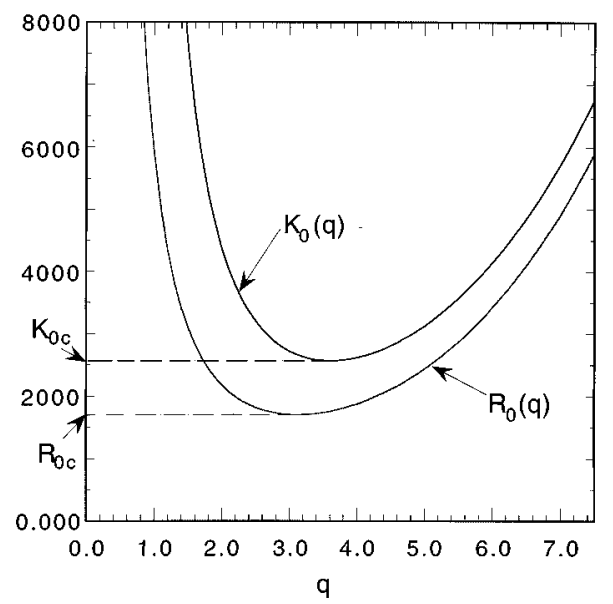

FIG. 1. Convective stability diagram for a horizontal layer of paramagnetic fluid in the presence of a vertical uniform magnetic field. Shown are the marginal Rayleigh number $R_{0}(q)$ for the onset of convection in the absence of magnetic fields $(K=0)$ and the marginal Kelvin number $K_{0}(q)$ for the onset of convection in the absence of gravity $(R=0)$, as a function of the wave number $q$. Also indicated are the corresponding critical values for the onset of convection, $R_{0 c}=1707.762$ and $K_{0 c}=2568.476$.

For transverse rolls, the circulating convective flow of fluid elements tends to misalign the magnetic moments from the field direction. The magnetic torque must do work to ensure continued alignment with the field; the magnetization $\mathbf{M}$ $=\chi \mathbf{H}$ remains parallel to the field. Such work might tend to suppress transverse rolls in much the same way that dissipative effects suppress buoyancy-driven convection. Before transverse rolls are possible, the driving force must be strong enough to overcome not only the viscous dissipation, but also the tendency of the magnetic moments to align with the field. Therefore, the field would tend to suppress transverse rolls. Nevertheless, the circulating convective flow of longitudinal rolls does not misalign the magnetic moments from the field direction, and the field thus has no effect on longitudinal rolls, as indicated by $K^{\prime}=0$.

A nontrivial solution requires a vanishing determinant of the coefficient matrix in Eq. (18), yielding the generalized marginal condition relating the Rayleigh number $R$, the reduced Kelvin number $K^{\prime}$, and the dimensionless wave number $q$. To obtain this condition numerically, we truncate the infinite series in Eq. (18) to a finite number $N$ of terms. For given values of $N, q$, and $K^{\prime}$, we adjust $R$ numerically until the determinant vanishes. This procedure yields the marginal Rayleigh number $R=R\left(q, K^{\prime}\right)$, which can be minimized with respect to $q$ to obtain the critical Rayleigh number $R_{c}$ for given $K^{\prime}$.

For the classical, nonmagnetic case with $K^{\prime}=0$ this procedure yields the known marginal Rayleigh number $R$ $=R_{0}(q)$ in the absence of magnetic fields. Minimizing $R_{0}(q)$ with respect to $q$ for $N=5,10$, and 15 yields the successive estimates $R_{0 c}=1707.784,1707.763$, and 1707.762 , with $q_{0 c}=3.116$ in each case. These rapidly converge to the well known critical values ${ }^{1} R_{0 c}=1707.762$ and $q_{0 c}=3.117$. Figure 1 shows $R_{0}(q)$ for the 15 -term truncation, which is used henceforth. A static fluid layer in the absence of magnetic fields is stable to convective perturba-

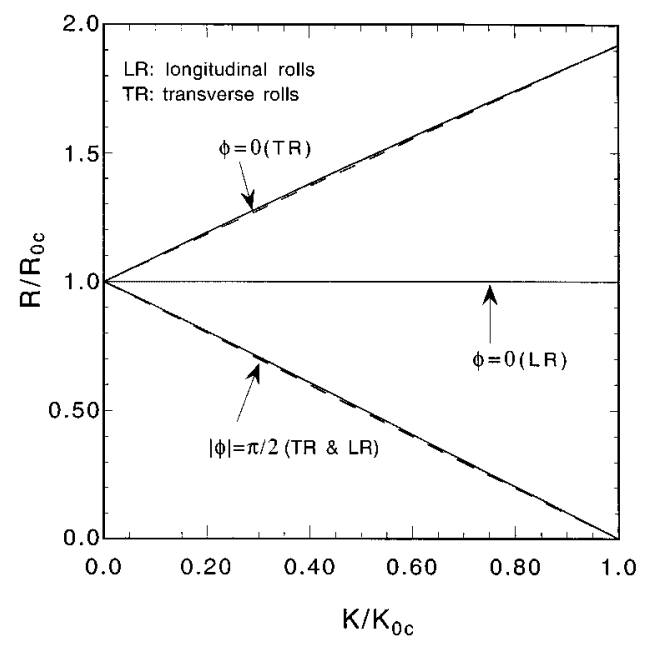

FIG. 2. Phase diagram for the convective threshold, giving the ratio $R_{c} / R_{0 c}$ of the critical Rayleigh number $R_{c}$ from our simulations (dark trace) to the classical nonmagnetic critical Rayleigh number $R_{0 c}=1707.762$ as a function of the ratio $K / K_{0 c}$ of the Kelvin number to the critical Kelvin number $K_{0 c}=2568.476$ for vertical fields and no gravity. Traces are shown for $\phi=0$ (horizontal fields) and $\phi= \pm \pi / 2$ (upward and downward fields). All points below the trace appropriate for a particular value of $\phi$ are stable to the appropriate (longitudinal or transverse) rolls, whereas all points above this trace (for larger $R$ ) are unstable to these rolls. Vertical fields with $\phi$ $= \pm \pi / 2$ render $R_{c}<R_{0 c}$ for $K>0$, and thereby promote convection. For horizontal fields $(\phi=0), R=R_{c}$ for all $K$ for longitudinal rolls, reflecting that the magnetic field has no effect on these rolls; whereas $R / R_{c}$ increases with increasing $K / K_{0 c}$ for transverse rolls, indicating that the magnetic field suppresses these rolls. Dashed traces give approximate linear results from Eq. (21).

tions for Rayleigh numbers $R<R_{0 c}$, above which gravitational buoyancy destabilizes a band of wave numbers centered approximately on $q_{0 c}$.

A uniform vertical magnetic field $(\phi= \pm \pi / 2)$ yields a maximum, $\xi$-independent reduced Kelvin number $K^{\prime}=K$. To study the effect of this field on the layer, we first set $R$ $=0$ in Eq. (19) to obtain the marginal Kelvin number $K$ $=K_{0}(q)$ in the absence of gravity (Fig. 1), whose interpretation is similar to $R_{0}(q)$. Thus, the susceptibility gradient resulting from the imposed thermal gradient plays a role similar to buoyancy in destabilizing the layer, as expected. Minimizing $K_{0}(q)$ with respect to $q$ yields the critical values $K_{0 c}=2568.476$ and $q_{c}=3.609$, which implies that magnetically induced convection occurs for $K \geqslant K_{0 c}$. The form of the convection at marginal stability is not determined, reflecting the rotational symmetry of the layer with respect to the field. However, for an oblique field directed at an angle $\phi$ $(|\phi|<\pi / 2)$ with respect to the horizontal, the layer is no longer rotationally symmetric about the vertical, and the form of the convection can be determined. Since only the reduced Kelvin number $K^{\prime}=K\left(\sin ^{2} \phi-\cos ^{2} \phi \cos ^{2} \xi\right)$, appears in Eq. (19) and since longitudinal rolls are the most unstable rolls, magnetically induced convection appearing as longitudinal rolls occurs for $K \sin ^{2} \phi \geqslant K_{0 c}$. There is no convection for horizontal magnetic fields in the absence of gravity, as expected.

Including both gravitational buoyancy and magnetic fields, Fig. 2 shows the critical Rayleigh number $R_{c}$ (dark traces) versus the Kelvin number $K$, scaled, respectively, by 
the values $R_{0 c}=1707.762$ and $K_{0 c}=2568.476$ discussed above. Traces are shown only for $\phi=0$ (horizontal fields) and $\phi= \pm \pi / 2$ (upward and downward fields), the primary examples. All points below the trace for the appropriate value of $\phi$ are stable to the appropriate (longitudinal or transverse) rolls, whereas all points above this trace are unstable to these rolls. For example, the horizontal trace for $\phi=0$ (LR) simply indicates that $R_{c}=R_{0 c}=1707.762$ for all $K$ for the onset of longitudinal rolls, reflecting the absence of any magnetic effects on these rolls for fields directed horizontally. Vertical magnetic fields parallel or antiparallel to gravity (with $\phi= \pm \pi / 2$ ) render $R_{c}$ less than $R_{0 c}$ for $K>0$, and thereby destabilize the static layer to convective rolls of ar- bitrary orientation. As the strength of the vertical magnetic field increases with increasing $K$, the ratio $R_{c} / R_{0 c}$ decreases. When the Kelvin number $K$ reaches its critical value $K_{0 c}$ for the onset of convection in the absence of gravity, $R_{c}$ falls to zero as expected. The trace for $\phi=0$ (TR) implies that $R_{c}$ is greater than $R_{0 c}$ for $K>0$, indicating that a horizontal magnetic field inhibits transverse rolls. The larger the Kelvin number $K$, the larger the critical Rayleigh number $R_{c}$ for the onset of these transverse rolls, and the more difficult it is to drive them.

An approximate linear relation between $R_{c} / R_{0 c}$ and $K / K_{0 c}$ follows from fits to the end points in Fig. 2:

$$
\frac{R_{c}}{R_{0 c}}= \begin{cases}1-\frac{K}{K_{0 c}}\left(\sin ^{2} \phi-\cos ^{2} \phi \cos ^{2} \xi\right), & \text { for } \sin ^{2} \phi \geqslant \cos ^{2} \phi \cos ^{2} \zeta \\ 1-0.924 \frac{K}{K_{0 c}}\left(\sin ^{2} \phi-\cos ^{2} \phi \cos ^{2} \xi\right), & \text { for } \sin ^{2} \phi<\cos ^{2} \phi \cos ^{2} \xi,\end{cases}
$$

where $R_{0 c}=1707.762$ and $K_{0 c}=2568.476$, as before. In the presence of a uniform oblique magnetic field, Eq. (21) yields the critical Rayleigh number $R_{c}$ for the onset of linear two-dimensional convective rolls in the $x-z$ plane. This relation gives $R_{c}$ to within $1 \%$ over the entire parameter range (see the dash traces in Fig. 2 for $\phi=0$ and $|\phi|=\pi / 2$ ). Furthermore, they are valid for all angles $\phi$ and all angles $\xi$. This relation reduces to the result obtained by Finlayson ${ }^{6}$ for a vertical field $(\phi$ $=\pi / 2$ ). Equation (21) shows that longitudinal rolls are the most unstable ones. It also shows that transverse rolls are the most difficult ones to drive.

Our numerical calculation also reveals an approximate linear relation for the critical wave number $q_{c}$ as a function of $K, \phi$, and $\xi$ :

$q_{c}= \begin{cases}q_{0 c}+1.92 \times 10^{-4} K\left(\sin ^{2} \phi-\cos ^{2} \phi \cos ^{2} \xi\right), & \text { for } \sin ^{2} \phi \geqslant \cos ^{2} \phi \cos ^{2} \xi, \\ q_{0 c}+1.97 \times 10^{-4} K\left(\sin ^{2} \phi-\cos ^{2} \phi \cos ^{2} \xi\right), & \text { for } \sin ^{2} \phi<\cos ^{2} \phi \cos ^{2} \xi,\end{cases}$

where $q_{0 c}=3.116$, the critical wave number in the absence of magnetic fields. This relation gives the critical wave number $q_{c}$ for the onset of the rolls to within $1 \%$ of its true value for $K \leqslant K_{0 c}$. For longitudinal rolls ( $\xi=\pi / 2$ ), Eq. (22) shows that the critical wave number $q_{c}>q_{0 c}$ for $|\phi|>0$. Thus, the critical wavelength $\lambda_{c}=2 \pi / q_{c}$ is smaller than the critical wavelength $\lambda_{0 c}=2 \pi / q_{0 c}$ in the absence of magnetic fields, indicating that the oblique field tends to narrow the longitudinal rolls. Equation (22) also reveals that the larger the Kelvin number $K$, the smaller the critical wavelength $\lambda_{c}$ for given $\phi$. For transverse rolls $(\xi=0)$, Eq. (22) yields $q_{c}$ $>q_{0 c}$ for $|\phi|>\pi / 4$ and $q_{c}<q_{0 c}$ for $|\phi|<\pi / 4$, indicating that the field tends to narrow the transverse rolls when $|\phi|$ $>\pi / 4$, but to widen them when $|\phi|<\pi / 4$. Therefore, horizontal magnetic fields have no effect on longitudinal rolls, but widen transverse rolls.

In summary, Eqs. (21) and (22) give the predicted critical Rayleigh number $R_{c}$ and critical wave number $q_{c}$ for the onset of the rolls in a vertical plane in a laterally unbounded horizontal layer of paramagnetic fluid that is heated from below and is subjected to a uniform oblique external magnetic field. These equations show that longitudinal rolls are the most unstable ones. Inserting $\xi=\pi / 2$ together with the angle $\phi$ and the Kelvin number,

$$
K=\frac{\mu_{0} \chi_{a}^{2} H_{0}^{2} \Delta T^{2} d^{2}}{\left(1+\chi_{a}\right) \rho_{a} T_{a}^{2} D_{T} \nu}
$$

relevant to a particular experiment into Eqs. (21) and (22) gives the predicted critical Rayleigh number $R_{c}$ and the critical wave number $q_{c}$ for the onset of longitudinal rolls for that experiment. If the Rayleigh number

$$
R=\frac{\alpha g \Delta T d^{3}}{D_{T} \nu}
$$

exceeds this critical value $R_{c}$, the static layer is predicted to be unstable to longitudinal rolls. Note that both $R$ and $K$ depend on the temperature difference $\Delta T$ across the layer.

Experimental tests of Eqs. (21) and (22) would be particularly welcome, especially those involving nonvertical magnetic fields. Paramagnetic fluids such as aqueous solutions of gadolinium nitrate ${ }^{7}$ or manganese chloride might make good candidates for experiments. The susceptibility of these solutions can be adjusted through the concentration of the paramagnetic salts. Typical values $\alpha=2.37 \times 10^{-4} \mathrm{~K}^{-1}$, $\nu=10^{-6} \mathrm{~m}^{2} / \mathrm{s}, \quad D_{T}=1.4 \times 10^{-6} \mathrm{~m}^{2} / \mathrm{s}$, and $d=6 \times 10^{-3} \mathrm{~m}$ for aqueous solutions imply a critical temperature difference $\Delta T_{c}=4.8 \mathrm{~K}$ for buoyancy-driven convection in the absence of magnetic fields in a terrestrial environment. Employing this temperature difference, together with the values $\chi$ 
$=10^{-3} \ll 1, \quad \rho_{a}=10^{3} \mathrm{~kg} / \mathrm{m}^{3}, \quad \mu_{0} H_{0}=8 \mathrm{~T}, \quad$ and $T_{a}=300 \mathrm{~K}$ yields a Kelvin number $K \approx 335$, which is about $13 \%$ of the critical value $K_{0 c}=2568.476$. These values might allow the exploration of the magnetic effects on convection. The measured relation between the Nusselt number, the ratio of the heat transport with and without convection, and the temperature difference $\Delta T$ can be used to determine $R_{c}$. Experimental procedures for examining the magnetic effect on convection might include explorations of the critical Rayleigh number $R_{c}$ or the Nusselt number as a function of $\phi, K$, or $\Delta T$. Analysis of the basic flow patterns might be accomplished without visualization by measuring the magnetic field in a plane just above or below the layer, which would reflect the convective flow pattern.

In conclusion, our linear stability analysis of a horizontal paramagnetic fluid layer heated from below in the presence of a uniform oblique magnetic field predicts that longitudinal rolls are the most unstable ones. Inclined uniform magnetic fields have the maximum enhancing effect on longitudinal rolls, and tend to narrow these rolls as well. Horizontal fields have no effect on longitudinal rolls, but inhibit rolls in other directions. Experiments are solicited to test these predictions. Since convection can also be driven by external magnetic field gradients, ${ }^{7}$ continuing investigations ${ }^{8}$ are aimed at understanding the effect of such gradients.

\section{ACKNOWLEDGMENTS}

This research was supported by NASA under Grant No. NAG3-1921.

\section{APPENDIX: TEMPERATURE EQUATION}

The temperature equation for a magnetizable fluid placed in a magnetic field is derived by choosing the temperature $T$, the specific volume $v$, and the magnetic field $\mathbf{H}$ as the independent thermodynamic variables. ${ }^{6}$ This equation involves the specific heat capacity $c_{v, \mathbf{H}}$ of the fluid at constant volume and magnetic field. However, thermal expansion necessarily affects $v$, and practical experiments are generally performed at fixed pressure $p$. Here, we derive the temperature equation by choosing $T, p$, and $\mathbf{H}$ as the independent variables. The energy equation [(A1) in Ref. 6] is

$$
d u=T d s-p d v+\mu_{0} \mathbf{H} \cdot d \mathbf{I},
$$

where $u$ is the internal energy per unit mass, $s$ the entropy per unit mass, $\mathbf{I}=v \mathbf{M}$ the magnetic moment per unit mass, and $p$ the pressure including a magnetic contribution in addition to the usual thermodynamic pressure. The entropy equation [(A4) in Ref. 6] is

$$
\rho T \frac{d s}{d t}=-\nabla \cdot \mathbf{j}_{h}+\Phi,
$$

where $\mathbf{j}_{h}=-\kappa \boldsymbol{\nabla} T$ is the heat flux and $\Phi$ is the viscous dissipation.

Since

$$
d s=\left(\frac{\partial s}{\partial T}\right)_{p, \mathbf{H}} d T+\left(\frac{\partial s}{\partial p}\right)_{T, \mathbf{H}} d p+\left(\frac{\partial s}{\partial H_{i}}\right)_{T, p} d H_{i},
$$

we have

$$
\frac{d s}{d t}=\left(\frac{\partial s}{\partial T}\right)_{p, \mathbf{H}} \frac{d T}{d t}+\left(\frac{\partial s}{\partial p}\right)_{T, \mathbf{H}} \frac{d p}{d t}+\left(\frac{\partial s}{\partial H_{i}}\right)_{T, p} \frac{d H_{i}}{d t} .
$$

We rewrite Eq. (A1) as

$$
d \varphi=-s d T+v d p-\mu_{0} \mathbf{I} \cdot d \mathbf{H},
$$

where $\varphi=u-T s+p v-\mu_{0} \mathbf{I} \cdot \mathbf{H}$ is the Gibbs function. This equation requires

$$
\left(\frac{\partial s}{\partial p}\right)_{T, \mathbf{H}}=-\left(\frac{\partial v}{\partial T}\right)_{p, \mathbf{H}} \text { and }\left(\frac{\partial s}{\partial H_{i}}\right)_{T, p}=\mu_{0}\left(\frac{\partial I_{i}}{\partial T}\right)_{p, \mathbf{H}} .
$$

Substituting Eqs. (A2) and (A6) into Eq. (A4) yields

$$
\rho c_{p, \mathbf{H}} \frac{d T}{d t}-\alpha T \frac{d p}{d t}+\mu_{0} \rho T\left(\frac{\partial \mathbf{I}}{\partial T}\right)_{p, \mathbf{H}} \cdot \frac{d \mathbf{H}}{d t}=\kappa \nabla^{2} T+\Phi,
$$

where $c_{p, \mathbf{H}}=T(\partial s / \partial T)_{p, \mathbf{H}}$ is the specific heat capacity at constant pressure and magnetic field, and $\alpha=(1 / v)$ $\times(\partial v / \partial T)_{p, \mathbf{H}}$ is the thermal expansion coefficient.

For paramagnetic fluids, we have $\mathbf{M}=\chi \mathbf{H}=C \rho \mathbf{H} / T$ $=C \mathbf{H} / v T$, which yields $\rho T(\partial \mathbf{I} / \partial T)_{p, \mathbf{H}}=-\mathbf{M}$.

${ }^{1} \mathrm{~S}$. Chandrasekhar, Hydrodynamic and Hydromagnetic Stability (Dover, New York, 1981), Chap. 2 and Appendix V.

${ }^{2}$ M. C. Cross and P. C. Hohenberg, "Pattern formation outside of equilibrium," Rev. Mod. Phys. 65, 851 (1993).

${ }^{3}$ R. E. Rosensweig, Ferrohydrodynamics (Cambridge University Press, New York, 1985), Chap. 4.

${ }^{4}$ N. W. Ashcroft and N. D. Mermin, Solid State Physics (Saunders College, Philadelphia, 1976), Chap. 31.

${ }^{5}$ P. J. Stiles and M. Kagan, "Thermoconvective instability of a ferrofluid in a strong magnetic field," J. Colloid Interface Sci. 134, 435 (1990).

${ }^{6}$ B. A. Finlayson, "Convective instability of ferromagnetic fluids,' J. Fluid Mech. 40, 753 (1970).

${ }^{7}$ D. Braithwaite, E. Beaugnon, and R. Tournier, "Magnetically controlled convection in a paramagnetic fluid," Nature 354, 134 (1991).

${ }^{8}$ J. Huang, B. F. Edwards, and D. D. Gray, "Magnetic control of convection in nonconducting paramagnetic fluids," submitted to Nature. 